\title{
Dreidimensionale C-Bogen-Darstellung im Experiment und Klinik
}

\author{
Ekkehard Euler, Sandro Michael Heining, Tanja Fischer, Rock Clemens, \\ Dorothea Kotsianos, Ulrich Linsenmaier, Klaus-Jürgen Pfeifer, Wolf Mutschler
}

\section{Zusammenfassung}

Mit dem C-Bogen Siremobil Iso-C $C^{3 D}$ (Siemens AG, Erlangen) ist zusätzlich zur herkömmlichen Durchleuchtung die dreidimensionale Darstellung von Hochkontrastobjekten wie menschlichem Knochen in situ möglich. Der prinzipielle Unterschied zu herkömmlichen C-Bögen ist das isozentrische Design in Verbindung mit einem Motorantrieb zur automatischen Orbitalbewegung und einer computerunterstützten Bildbearbeitung. In einer Serie experimenteller Studien an anatomischen Leichenpräparaten konnte gezeigt werden, dass die Anwendung an kleinen Extremitätengelenken eine ausreichende diagnostische Sicherheit bietet, die mit der Computertomographie (CT) vergleichbar ist. Für die intraoperative Kontrolle rekonstruierter Gelenkflächen und eingebrachten Osteosynthesematerials, wie z.B. Osteosyntheseschrauben in Gelenknähe, ist dieses
Verfahren aussagekräftiger als die intraoperative Durchleuchtung [1]. Seit der Zulassung des Gerätes in der zweiten Hälfte des letzten Jahres hat es sich im klinischen Einsatz bereits sehr bewährt. Die Erfahrungen bestätigen die Erwartung eines hohen intraoperativen Bildinformationsgewinns. Auch zeichnen sich Einsparungen von post-, aber auch präoperativen CT's ab. Die Darstellung rumpfnaher Gelenke und der Wirbelsäule verspricht nach unseren bisherigen experimentellen, präklinischen Erfahrungen ebenfalls einen intraoperativen Informationsgewinn, der besonders in der Kombination mit einem Navigationssystem deutlich wird. Die Bedienung und der Raumbedarf des Gerätes ist vergleichbar mit herkömmlichen CBögen, so dass eine Umstrukturierung des Operationssaals oder der Arbeitsabläufe nicht erforderlich ist. Metallfreie Lagerungsflächen zur Vermeidung von Metallartefakten sind allerdings notwendig.

\section{Einführung}

Bei unfallchirurgischen und orthopädischen Operationen ist für ein optimales Behandlungsresultat u.a. die Rekonstruktion von Gelenkflächen sowie die korrekte Lage von eingebrachtem Osteosynthesematerial (besonders Osteosyntheseschrauben in Gelenknähe oder in der Nähe gefährdeter vaskulärer oder nervaler Strukturen) essenziell. Zur Lagekon-

OP-JOURNAL 2002; 17: 46-50

(c) Georg Thieme Verlag Stuttgart · New York trolle von Frakturfragmenten, von Implantaten (z.B. Osteosyntheseplatten, -schrauben oder -nägeln) oder anderer technischer Hilfsmittel (z.B. Führungsdrähte) wird im Allgemeinen die intraoperative Durchleuchtung mit C-Bogen gewählt. Hierbei ist die Erkennung bzw. Zuordnung der räumlichen, dreidimensionalen Anordnung der dargestellten Objekte (Knochen, Implantate) im zweidimensionalen Durchleuchtungsbild oft schwierig. Ein unbefriedigendes Rekonstruktionsergebnis bei dislozierten Gelenkfrakturen oder eine Schraubenfehllage werden daher vielleicht erst in einer postoperativen Kontroll-Computertomographie (CT) sichtbar. In der Wirbelsäulenchirurgie wurden deshalb für die Pedi-
kelschrauben-Platzierung Navigationssysteme auf der Basis präoperativ angefertigter CT-Datensätze entwickelt [5], mit deren Hilfe die Rate der Fehlplatzierung erheblich gesenkt werden konnte [3]. Bei diesen Systemen wird, vergleichbar den in der KFZ-Industrie realisierten GPS-Navigationssystemen, die Position des chirurgischen Instruments (KFZGPS: Fahrzeug) über ein Kamerasystem (KFZ-GPS: Satelliten) in das präoperative CT-Bild oder das intraoperative C-BogenBild (KFZ-GPS: Stadtplan, Landkarte) übertragen (virtuelle Realität). In der rekonstruktiven Chirurgie von Gelenkfrakturen gestatten diese Systeme jedoch keine exakte intraoperative Kontrolle über das Repositionsergebnis, da die Art und die Anzahl der mit dem Navigationssystem erfassbaren Knochenfragmente derzeit auf wenige große Fragmente limitiert ist.

Eine neue Methode der intraoperativen 3D-Bildgebung mit einem mobilen isozentrischen C-Bogen (Siremobil Iso- $\mathrm{C}^{3 \mathrm{D}}$, Siemens AG, Erlangen; Abb.1) erlaubt es erstmals, die intraoperativ routinemäßig praktizierte C-Bogen-Anwendung mit den Vorteilen einer intraoperativen 3DDarstellung bei einem im Vergleich zum bisherigen Vorgehen (Bildwandler und prä- bzw. postoperatives CT bzw. intraoperatives $\mathrm{CT}$ ) wesentlich geringeren Aufwand zu kombinieren. Das neue System ermöglicht es, während einer automatisierten kontinuierlichen orbitalen Rotation um $190^{\circ}$ eine feste Zahl von Durchleuchtungsbildern (100 oder 50 Einzelbilder) aufzunehmen. Der angeschlossene Computer errechnet daraus simultan einen hochaufgelösten dreidimensionalen Bildwürfel von etwa $12 \mathrm{~cm}$ Kantenlänge. Durch die integrierte Bildbearbeitung und die Bedieneroberfläche kann sich der Untersucher Schnittbilder in jeder beliebigen Schnittebene erzeugen (Abb. 2 u.3). Durch weitere Bildbearbeitung ist eine Oberflächenrekonstruktion aus dem erzeugten Datensatz ebenfalls möglich (Abb.4). 

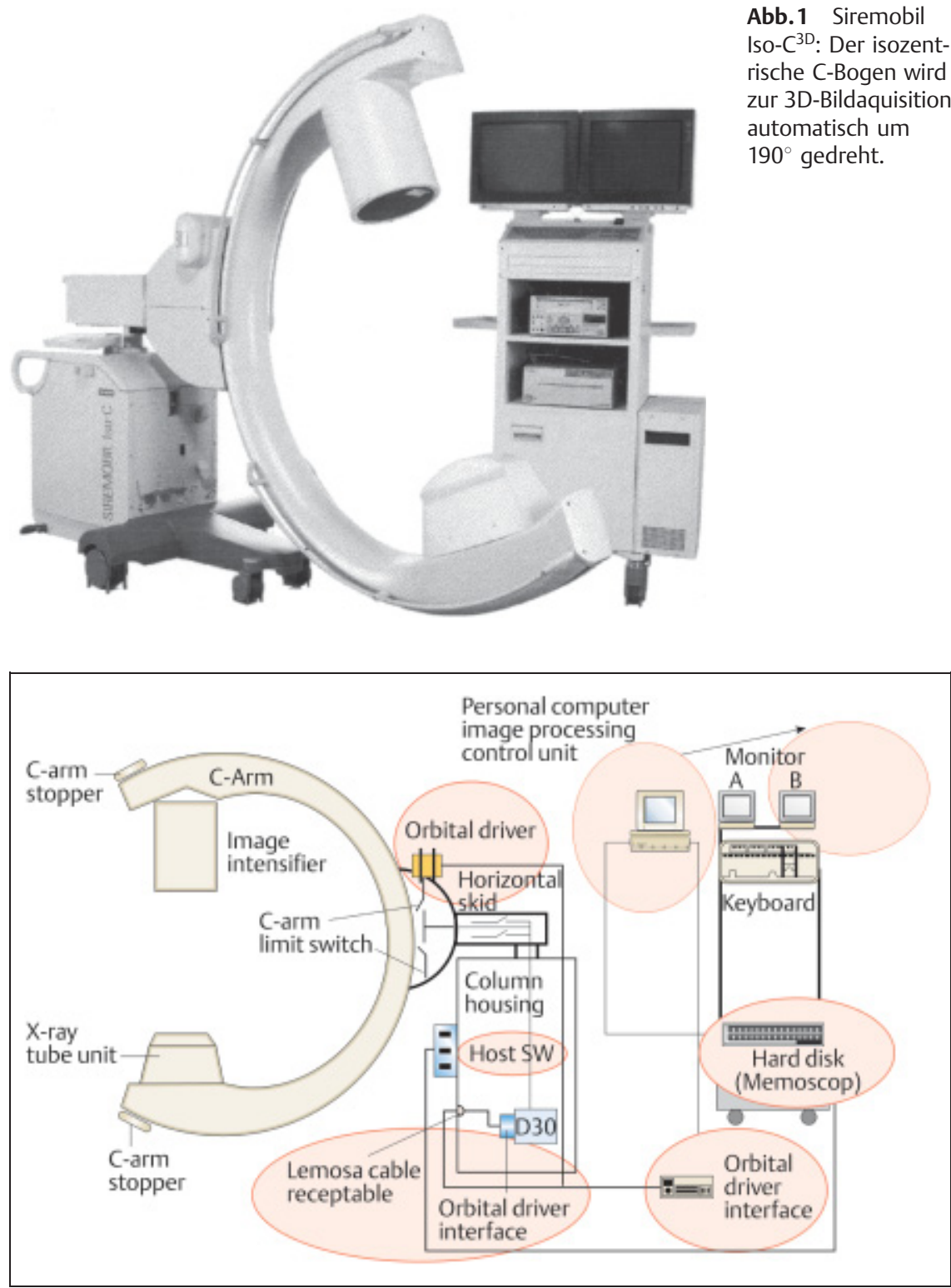

Abb. 2 Schema der Bilddatenverarbeitung des Siremobil Iso-C ${ }^{3 D}$ (Quelle: Siemens AG).

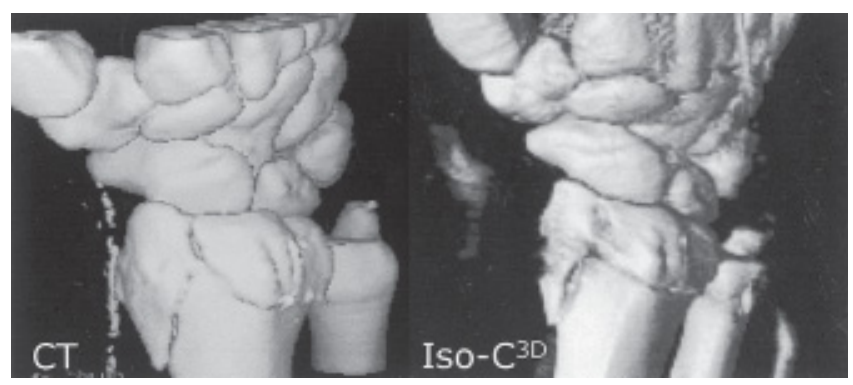

Abb. 4 Vergleich der Oberflächenrekonstruktionen aus CT- und Siremobil Iso-C ${ }^{3 \mathrm{D}}$-Datensätzen am Beispiel einer distalen Radiusfraktur.

Mit dem isozentrischen C-Bogen Iso- $\mathrm{C}^{3 \mathrm{D}}$ können Schnittbilder in jeder beliebigen Schnittebene hergestellt werden.

In der vorliegenden Arbeit wird der hohe diagnostische Wert der dreidimensionalen Bildrekonstruktion für die intraopera-

Abb. 1 Siremobil Iso-C ${ }^{3 \mathrm{D}}$ : Der isozentrische C-Bogen wird automatisch um $190^{\circ}$ gedreht. ten am Extremitätenskelett anhand ger Studien $[1,2,5,6]$ aufgezeigt. In diesen Studien wurden die Bildqualität und die Strahlendosis, die Frakturerkennbarkeit und die diagnostische Aussagekraft am Osteosynthesemodell im Vergleich mit

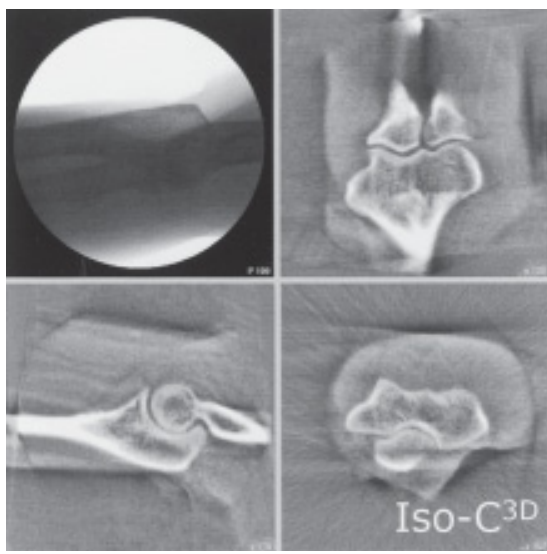

Abb. 3 Darstellung eines der 100 Durchleuchtungsbilder (links oben) sowie der drei unterschiedlichen Schnittebenen am Beispiel eines Ellbogengelenkes.

anderen Verfahren untersucht. Bis zur Klinikeinführung des Gerätes war auch die Frage zu klären, ob sich besondere Anforderungen an die Lagerungstechniken und die Handhabung im sterilen Umfeld ergeben. Bei der praktischen Anwendung interessierte, ob durch die neue Technik ein Einfluss auf den intraoperativen Work-Flow bei Osteosynthesen festgestellt werden kann. Schließlich werden erste Erfahrungen mit reellen Frakturversorgungen geschildert.

\section{Material, Methode}

Mit einem Prototyp des Siremobil Iso$\mathrm{C}^{3 \mathrm{D}}$-Bildwandlergerätes wurden die Hochkontrastauflösung ermittelt und Dosismessungen vorgenommen $[2,5]$. Weiterhin wurden an verstorbenen Unfallopfern und anatomischen Leichen dreidimensionale C-Bogen-Untersuchungen und vergleichende Spiral-CT-Untersuchungen der Extremitätengelenke durchgeführt. Die Fragestellungen bezogen sich hierbei auf die Erkennung von anatomischen Strukturen und Frakturen, auf den Einfluss von Metallartefakten, auf die diagnostische Aussagekraft der Schnittbilder sowie auf die Anforderungen an die Lagerungstechnik [1,2]. Als Untersuchungsliege diente eine $40 \mathrm{~cm}$ breite Carbonfaser-Platte (Rückenplatte für Neurochirurgie, System 1150; Maquet AG, Rastatt), um die bei üblichen Untersuchungsliegen vorhandenen Metallartefakte auszuschalten.
Seit der Zulassung des Gerätes für die klinische Anwendung an HWS und Gelenken der Extremitäten wurden die präklinischen Erkenntnisse in die OP-Praxis 

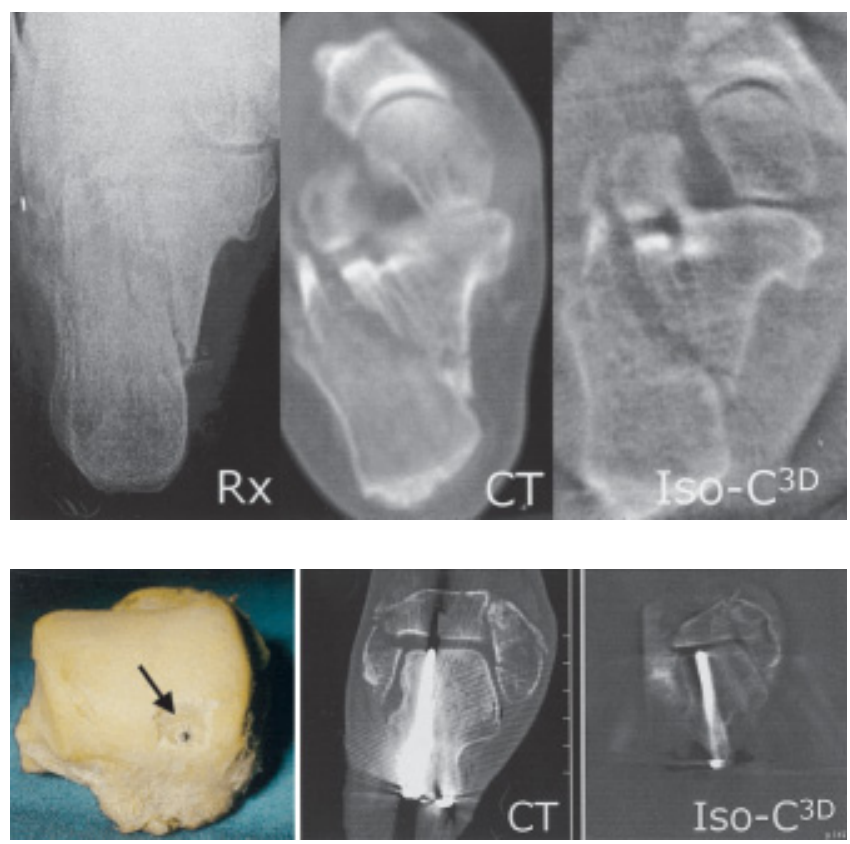

Abb. 6 Untersuchung zur Erkennbarkeit von Schrauben(fehl)lagen bei Osteosynthesen am Talus-Schraubenosteosynthese-Modell: Im Iso-C $\mathrm{C}^{3 \mathrm{D}}$ Bild ist die intraartikuläre Fehllage ebenso sicher zu erkennen wie im CTBild.

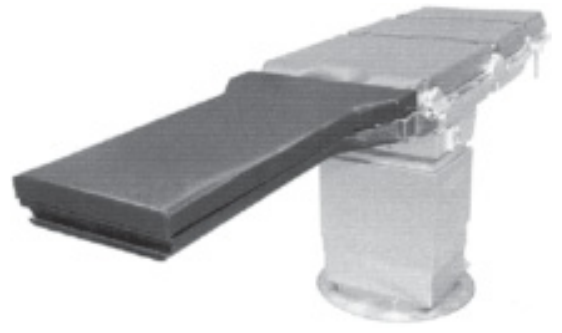

Abb. 7 Röntgentransparente Patientenliege aus Carbonfaser-Gewebe (Quelle: Maquet, Rastatt).

übertragen und der Iso-C $\mathrm{C}^{3 \mathrm{D}}$-Bildwandler im täglichen Routinebetrieb eingesetzt.

\section{Ergebnisse}

Die Hochkontraststudien zur Ermittlung der Bildauflösung zeigten mit einer Hochkontrastauflösung von $9 \mathrm{LP} / \mathrm{cm}$ eine der CT vergleichbare Detailerkennbarkeit [5]. Die Strahlendosen (Dosislängenprodukte, Flächendosisprodukte) des Iso- $\mathrm{C}^{3 \mathrm{D}}$ waren bei Verwendung der Standardeinstellung für kleine Extremitätengelenke (100 Einzelbilder) mit denen einer Spiral-CT vergleichbar, bei der Einstellung für große Gelenke lag sie höher. Auch muss bei Untersuchungen des Körperstamms mit einer nicht unerheblichen Streustrahlung gerechnet werden [6]. Frakturen in Gelenknähe (Abb.5) konnten mit den Iso- $\mathrm{C}^{3 \mathrm{D}}$-Bildern ebenso sicher
Abb. 5 Vergleichende Darstellung einer Kalkaneusfraktur im konventionellen Röntgenbild (Rx), $\mathrm{CT}$ und Iso- $\mathrm{C}^{3 \mathrm{D}}$.

wie mit CT-Bildern und signifikant besser als mit konventionellen Röntgenbildern diagnostiziert werden [2]. Auch am Schrauben-Osteosynthesemodell des Talus (Abb.6) konnte die Schraubenlage (intraossär - intraartikulär) mit dem Iso- $\mathrm{C}^{3 \mathrm{D}}$ und dem $\mathrm{CT}$ gleich gut und signifikant besser als mit der Durchleuchtung oder dem konventionellen Röntgenbild erkannt werden [1].

Frakturen und Schrauben(fehl)lagen können im Iso-C ${ }^{3 \mathrm{D}}$-Bild ebenso gut wie im CT-Bild erkannt werden.

Die klinische, intraoperative Anwendung des C-Bogens zur dreidimensionalen Darstellung der HWS sowie der unteren und oberen Extremität ist bei Verwendung metallfreier Unterlagen unproblematisch (Abb. 7).

Röntgentransparente Patientenliegen sind erforderlich.

Die neue Bildgebung wurde bisher bei gut 30 Patienten zur Frakturversorgung (Tab.1) angewendet. Der jetzt etablierte Work-Flow lässt sich wie folgt skizzieren: Fragmentreposition und präliminäre Fixierung durch Kirschner-Drähte unter konventioneller 2D-Bildwandler-Durchleuchtung, Kontrolle in der 3D-Rekonstruktion („präliminärer Scan“), definitive Osteosynthese 2D-kontrolliert, ab-

schließende 3D-Rekonstruktion („definitiver Scan“). Jeweils unmittelbar anschließend erfolgt die Bearbeitung der Schnittbilder (Fensterung, Wahl der Schnittebenen) vorzugsweise durch den Operateur selbst. Rekonstruktionen in den anatomischen Ebenen sowie in den Ebenen der Implantate haben sich bewährt. So können z.B. im präliminären Scan das erreichte Repositionsergebnis oder die Lage von Kirschner-Drähten kontrolliert oder die Länge der einzubringenden Schrauben bestimmt werden. Die Speicherung eines jeden Scans im DicomFormat erfolgt automatisch, es ist jedoch ratsam, im selben Arbeitsgang wichtige einzelne Schnittbilder zu Dokumentationszwecken sofort zu speichern. In der überwiegenden Zahl der Fälle wurden zwei Scans durchgeführt, wobei meist die abschließende 3D-Bildgebung ein postoperatives $\mathrm{CT}$ ersetzte.

Relevante Schnittbilder sofort speichern!

Beispiele der 3D-Darstellungen sind in den Bilddokumenten dargestellt und in den Abbildungslegenden (Abb. 8-11) beschrieben.

\section{Diskussion}

Mit der bislang vorliegenden experimentellen und klinischen Erfahrung kann der neuen Technik dreidimensionaler Bilderzeugung mit dem Siremobil Iso- $\mathrm{C}^{3 \mathrm{D}}$ ein hoher Stellenwert bei der Frakturversorgung an Extremitäten und Halswirbelsäule zugesprochen werden. Die Größe und die Handhabung des Gerätes unterscheiden sich kaum von den bisher üblichen C-Bögen und erfordern daher keinen wesentlichen logistischen Mehraufwand. Die Strahlenbelastung bei der Durchführung eines Scans mit 100 Einzelbildern am Rumpfskelett erfordert eine entsprechende, kritische Indikationsstellung. In der Praxis hat sich erwiesen, dass an den Extremitätengelenken (Hand-, Ellenbogen-, Sprung- und Kniegelenk) für traumatologische Frage-

Tab. 1 Klinischer Einsatz des Iso- $\mathrm{C}^{3 \mathrm{D}}$ in der Frakturversorgung.

$\begin{array}{lr}\text { HWS } & 3 \\ \text { Ellbogen } & 5 \\ \text { Handwurzel, Mittelhand } & 2 \\ \text { Handgelenk } & 8 \\ \text { Kniegelenk } & 4 \\ \text { Pilon, OSG } & 3 \\ \text { Fußwurzel } & 7 \\ \text { Summe } & 32\end{array}$




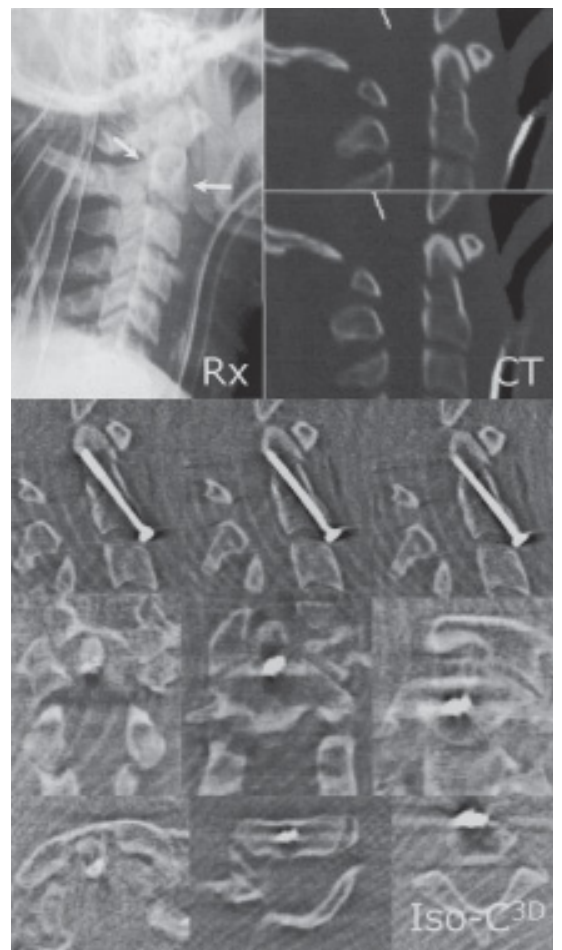

Abb. 8 Präoperative konventionelle (Rx) und computertomographische (CT) Abbildungen einer Dens-Fraktur; die intraoperativen IsoC $^{3 D}$-Schichtbilder (Iso-C ${ }^{3 D}$ ) lassen die korrekte Lage der beiden Osteosyntheseschrauben erkennen.

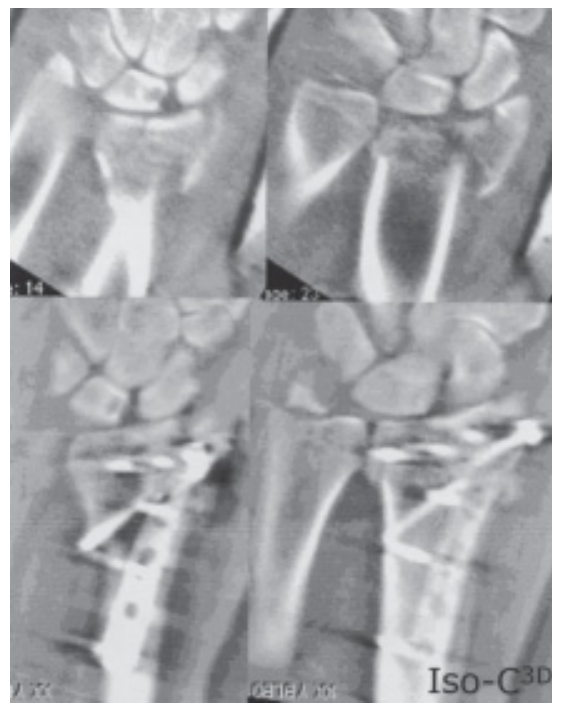

Abb.9 Distale Radiusfraktur in der dreidimensionalen Iso-C ${ }^{3 \mathrm{D}}$-Bildgebung vor und nach Plattenosteosynthese.

stellungen (Rekonstruktion von Gelenkflächen, Lage von Osteosynthesematerialien) 50 Einzelbilder anstelle von 100 Einzelbildern für die dreidimensionale Rekonstruktion und die intraoperative Diagnostik ausreichen. Wegen der nicht

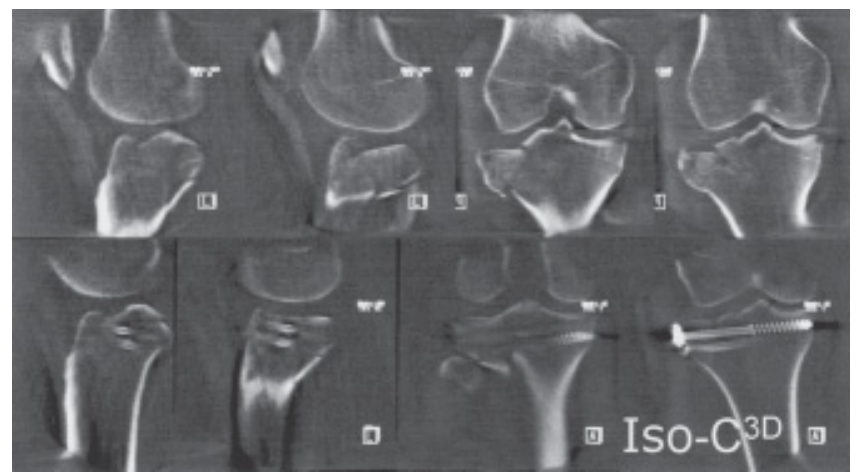

Abb.10 Tibiakopffraktur: Intraoperative Schichtbilder vor (oben) und nach (unten) Schraubenosteosynthese.

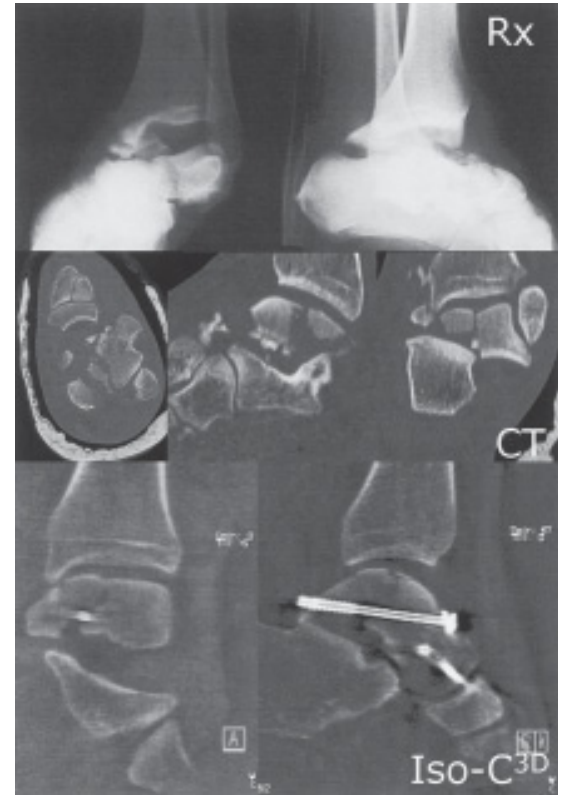

Abb.11 Talus-Luxations-Fraktur im konventionellen Röntgenbild (Rx) und im präoperativen Computertomogramm (CT); die intraoperativen Iso- $\mathrm{C}^{3 \mathrm{D}}$-Bilder (Iso- $\mathrm{C}^{3 \mathrm{D}}$ ) zeigen die wiederhergestellte Gelenkfläche und die gute Schraubenlage.

unerheblichen Streustrahlung sind die Strahlenschutzbestimmungen beim 3DBetrieb im Operationssaal (Röntgenschürzen, Abstand zur Strahlenquelle) unbedingt einzuhalten.

Wie bei allen Strahlenquellen: Strahlenschutzbestimmungen beachten!

Die dreidimensionale Bildgebung verleiht jedenfalls intraoperativ die diagnostische Sicherheit einer optimalen Beurteilung von Gelenkflächen und Implantatlagen. Eine besondere Bedeutung wird die Methode daher bei der Rekonstruktion von Gelenkflächen und auf dem Gebiet minimal-invasiver Instrumentierungen bei Eingriffen an peripheren Extremitätengelenken erlangen.

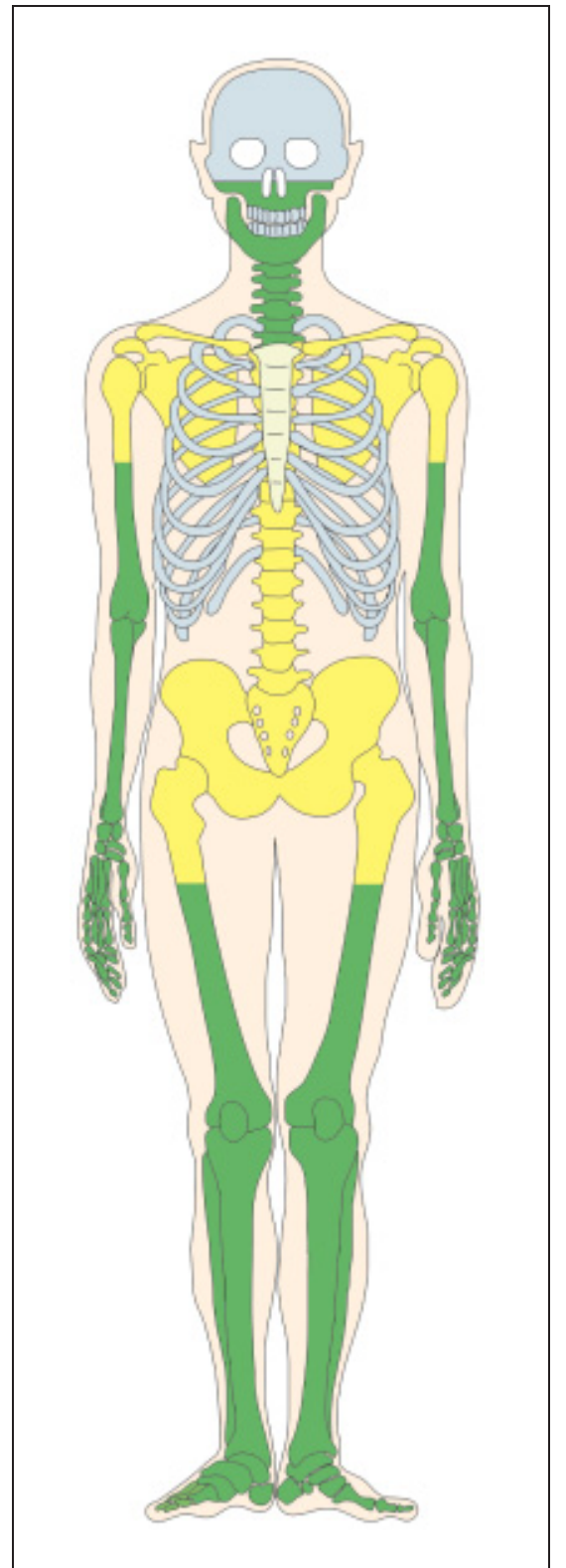

Abb.12 Einsatzbereiche der Iso- $\mathrm{C}^{3 \mathrm{D}}$-Technologie in der Praxis: grün: gute Einsatzmöglichkeiten; gelb: eingeschränkte Einsatzmöglichkeiten. 
Die 3D-Bildgebung mit dem Iso- $\mathrm{C}^{3 \mathrm{D}}$ ist besonders bei der Rekonstruktion peripherer Extremitätengelenke hilfreich.

Wir sehen jedoch auch ein Potential bei der Darstellung zentraler Extremitätengelenke und des Stammskeletts (Abb.12). In der Zukunft wird die C-Bogen-basierte 3D-Bildgebung in Kombination mit anderen technischen Verfahren der Bildgebung und -auswertung, vor allem der Navigation, zum Einsatz kommen. Hierbei wird sich nicht nur die Möglichkeit einer Navigation in einem aktuellen (z.B. nach erfolgtem Repositionsmanöver) dreidimensionalen Datensatz, sondern auch die Möglichkeit der wesentlich einfacheren (nicht invasiven) Registrierung auch kleinerer freier Fragmente ergeben.

\section{Literatur}

${ }^{1}$ Euler E, Wirth S, Linsenmaier U, Mutschler W, Pfeifer KJ, Hebecker A. Vergleichende Untersuchung zur Qualität C-Bogen-basierter 3-D-Bildgebung am Talus. Unfallchirurg 2001: 104: 839-846
${ }^{2}$ Kotsianos D, Rock C, Euler E, Wirth S, Linsenmaier U, Brandl R, Mutschler W, Pfeifer KJ. 3D-Bildgebung an einem mobilen chirurgischen Bildverstärker (ISO-C-3D): Erste Bildbeispiele zur Frakturdiagnostik an peripheren Gelenken im Vergleich mit Spiral-CT und konventioneller Radiologie. Unfallchirurg 2001; 104: 834-838

${ }^{3}$ Laine T, Lund T, Ylikoski M, Lohikoski J, Schlenzka D. Accuracy of pedicle screw insertion with and without computer assistance: a randomised controlled clinical study in 100 consecutive patients. Eur Spine J 2000; 9: 235-40

${ }^{4}$ Nolte LP, Zamorano LJ, Jiang Z, Wang Q Langlotz F, Arm E, Visarius H. A Novel Approach to Computer Assisted Spine Surgery. Proc 1st Int Symp on Medical Robotics and Computer Assisted Surgery 1994; (MRCAS): $323-328$

5 Rock C, Linsenmaier U, Brandl R, Kotsianos D, Wirth S, Kaltschmidt R, Euler E, Mutschler W, Pfeifer KJ. Vorstellung eines neuen mobilen C-Bogen-/CT-Kombinationsgerät (ISO-C3D): Erste Ergebnisse der 3D-Schnittbildgebung. Unfallchirurg 2001; 104: 827-833

6 Rock C, Kotsianos D, Linsenmaier U, Fischer T, Brandl R, Vill F, Wirth S, Kaltschmidt R, Euler E, Pfeifer KJ, Reiser M. Untersuchungen zur Bildqualität, Hochkontrastauflösung und Dosis am Stamm- und Gliedmaßenskelett mit einem neuen dedizierten CT-System (ISO-C-3D) Fortschr Röntgenstr 2002; 174: $170-176$
Priv.-Doz. Dr. med. Ekkehard Euler

Oberarzt

Dr. med. Sandro Michael Heining

Assistenzarzt

Prof. Dr. med. Wolf Mutschler

Direktor

Chirurgische Klinik und Poliklinik

Klinikum der Universität

München-Innenstadt

Ludwig-Maximilians-Universität

Nussbaumstraße 20

D-80336 München

\section{Dr. med. Tanja Fischer}

Assistenzärztin

Dr. med. Dorothea Kotsianos

Assistenzärztin

Dr. med. Clemens Rock

Oberarzt

Dr. med. Ulrich Linsenmaier

Oberarzt

Dr. med. Klaus-Jürgen Pfeifer

Ltd. Oberarzt

Institut für Klinische Radiologie

Klinikum der Universität

München-Innenstadt

Ludwig-Maximilians-Universität

Nussbaumstraße 20

D-80336 München 